\title{
The effect of balneotherapy and peloid therapy on changes in the functional state of patients with knee joint osteoarthritis: a randomized, controlled, single-blind pilot study
}

\author{
Lina Varzaityte $^{1}$ (D) - Raimondas Kubilius ${ }^{1} \cdot$ Lolita Rapoliene $^{2} \cdot$ Ruta Bartuseviciute $^{3} \cdot$ Arvydas Balcius $^{3}$. \\ Kestutis Ramanauskas ${ }^{4} \cdot$ Irena Nedzelskiene $^{5}$
}

Received: 11 March 2019 / Revised: 9 August 2019 / Accepted: 11 August 2019 / Published online: 6 September 2019

(C) The Author(s) 2019

\begin{abstract}
The treatment of OA using pharmaceutical and non-pharmaceutical measures remains a topical subject. The purpose of this study is to assess the effect of natural factors (mineral water and mud) on changes in the functional state of patients with knee joint OA. Ninety-two adult people with grade I-III knee joint OA according to the Kellgren and Lawrence scoring system participated in the study. The subjects received 10 mineral water bath plus physical therapy or mud application procedures plus physical therapy or physical therapy alone every other day. The effectiveness of the treatment was assessed on the basis of anthropometric changes of data, VAS, SF-36, KOOS questionnaire indicators. Significantly greater walking speed, test of 5 sit downs/stand ups, circumference of a knee joint, flexion and extension range, flexor and extensor strength after treatment lasting 1 month were obtained in the intervention group. After 1 month after treatment pain intensity scores over the past month and when changing position were significantly higher in the control group. The positive changes in SF-36 were identified after 1 month after treatment: physical activity increased and pain decreased in the intervention groups. There was no significant difference between the averages of any KOOS subscale in groups. However, average percentages of symptoms, stiffness, and pain in the intervention groups were significantly better after treatment and lasting 1 month after treatment. Balneotherapy and peloid therapy effectively reduce pain and improve the functional state of patients with OA of a knee joint.
\end{abstract}

Keywords Knee joint osteoarthritis $\cdot$ Balneotherapy $\cdot$ Peloid therapy $\cdot$ Rehabilitation

\section{Introduction}

Knee joint osteoarthritis (OA) is a chronic degenerative knee joint disease, characterized by anatomical and/or

Lina Varzaityte

lina.varzaityte@gmail.com

1 Department of Rehabilitation, Lithuanian University of Health Sciences, A. Mickevičiaus str. 9, LT-44307 Kaunas, Lithuania

2 Department of Nursing, Klaipeda University, H. Mantas str. 84, LT-92294 Klaipeda, Lithuania

3 Limited company Medical SPA Eglès Sanatorija, Eglès str. 1, LT-66251 Druskininkai, Lithuania

4 Druskininkai Recreation and Health Centre, Vilniaus av. 11, LT-66119 Druskininkai, Lithuania

5 Department of Dental and Oral Pathology, Lithuanian University of Health Sciences, Mickevičiaus str. 9, LT-44307 Kaunas, Lithuania physiological disorders, which manifest in the degeneration of the joint cartilage, bone tissue rearrangement, formation of osteophytes, synovial membrane inflammation, joint capsule, and ligament damage as well as loss of normal function (Kraus et al. 2015). These processes lead to clinical symptoms of the disease - dull pain of aching nature or sharp, intermittent knee joint pain, crepitation, swelling as well as stiffness, decrease of movement amplitude, and leg muscle weakness (Anandkumar et al. 2014; Campbell et al. 2015). According to data from the World Health Organization (2013), in total, $9.6 \%$ of men and $18.0 \%$ of women older than 60 years of age suffer from symptomatic OA. Combination therapy is recommended for treatment of OA, using pharmaceutical and non-pharmaceutical measures at the same time, the purpose of which is to relieve pain, to slow down the progression of the disease and to improve or compensate for impaired movement function (Bruyère et al. 2014). The most commonly recommended non-pharmaceutical treatment measures are 
as follows: physiotherapy, weight correction, orthopedic or technical measures, electrical stimulation or treatment with other physical agents (Cutolo et al. 2015; McAlindon et al. 2014). Natural factors (balneotherapy or peloid therapy) have been referred to more and more in literature as treatment for musculoskeletal diseases (Karagülle and Karagülle 2015, Verhagen et al. 2015).

Balneotherapy traditionally means bathing in mineral and/ or thermal water from natural sources. On the other hand, treatment with gas molecules (e.g. $\mathrm{CO} 2, \mathrm{H}_{2} \mathrm{~S}$ ), mud or other natural factors in literature is often attributed to balneotherapy interventions (Fioravanti et al. 2017).

The aim of this study was to assess the effect of natural factors - mineral water and mud - on changes in the functional state of patients with knee joint OA.

\section{Materials and methods}

Clinical parameters (walking speed, time of 5 sit downs/stand ups, circumferences of thigh, knee, and calf, flexion/extension ranges, flexor/extensor strength) were assessed of all subjects during stage I (before treatment), stage II (after treatment), and stage III (after 1 month after treatment). Subjects' pain was assessed using visual analogue pain scale (VAS), where pain intensity is represented by a point between 0 and 10 (from "no pain" to "unbearable pain"). Subjects were assessed using life quality assessment questionnaire (SF-36v2 $®$ Health Survey Standard, Lithuania (Lithuanian)). It consists of 36 questions, which reflect eight sections of life: physical activity, restriction of activity due to physical problems, pain, overall health assessment, energy levels and vitality, social function, restriction of activity due to emotional disorders as well as emotional state. Answers to these questions are scored. Each section is scored from 0 to 100 using calculation algorithm. The higher the score, the better the quality of life. Distribution of validity of the scales of SF-36 questionnaire sections (Cronbach's alpha coefficient) at different stages of the study was as follows: at stage I -0.745 , at stage II -0.764 , at stage III -0.732 (The stability of the questionnaire over time is considered to be good enough when the interclass correlation coefficient is equal or greater than 0.70). Subjects were assessed using Knee injury and Osteoarthritis Outcome Score (KOOS) that is validated and adapted in Lithuania (Mapi research institute 2007). It is a subjective assessment method of functional state and quality of life in relation to the knee joint. It consists of 5 subscales - symptoms, stiffness, pain, mobility, everyday life and mobility, sports and recreational activities. The questionnaire was used for the assessment of respondents of all subjects during stage I (before treatment), stage II (after treatment), and stage III (after 1 month after treatment). On the basis of assessment methodology, presented on the official webpage of the questionnaire, assessments of each scale within a $100 \%$ system were obtained, where 100 means absence of symptoms, while 0 means significantly expressed symptoms. Validity of the questionnaire scale was also assessed. It was found that Cronbach's alpha of stage I was 0.959 , stage II -0.975 , and stage III -0.977 , i.e., strong internal scale consistency was demonstrated.

Statistical analysis was performed using Excel and SPSS 22. Descriptive statistical characteristics of data were calculated as follows: for quantitative data - the average and its standard deviation. Compliance of distribution of quantitative variables to normal distribution was tested using the Kolmogorov-Smirnov test.

The means of three groups were compared using the dispersion analysis (ANOVA). For multiple comparisons, Bonferroni test (post hoc) was applied. When the test of normality of the investigated variables was denied, KruskalWallis and the Mann-Whitney tests were used ( $F$-Fisher test, $\chi^{2}$-Kruskal-Wallis test). Between two dependent groups, non-parametric Wilcoxon signed ranks criteria were used. Interdependence of categorical variables was assessed by employing chi-square criteria. ROC (receiver operating characteristic) curve analysis method was used for determination of optimal values of parameters. The optimal values separated out the different groups with the highest accuracy. The logistic regression analysis was performed to determine odds ratio of participants with intervention group. Difference or relationship was considered to be statistically significant when the significance of the criteria was $p<0.05$.

For a probability to reasonably reject a false hypothesis, we calculated power of the study (when type 1 error $\alpha \leq 0.05$ ).

Power calculations were based on the article by Branco et al. (2016) "Bath thermal waters in the treatment of osteoarthritis of the knee: a randomized controlled clinical trial".

The results of the final study proved that $\mathrm{H} 0$ hypothesis was reasonably rejected, obtaining powers of the study $>0.9$ (pain on movement: baseline - 6.9 (2.1), after treatment -4.5 (2.5); pain at night: baseline - 4.2 (2.9), after treatment-2.4 (2.2); pain at rest: baseline -3.4 (2.4); after treatment-1.7 (1.7).

\section{Description of the research contingent}

This study was a randomized, controlled, single-blind comparison between balneotherapy plus physical therapy, mud therapy plus physical therapy, and physical therapy alone, which took place from $29 / 06 / 2018$ to $31 / 12 / 2018$, after obtaining Kaunas Regional Biomedical Research Ethics Committee permission (Nr. BE-2-65). All subjects signed the informed consent form prior to the initiation of the study. The inclusion criteria were as follows: subjects 18 years of age and older, with grade I-III knee joint OA according to the Kellgren and Lawrence scoring system participated in the 
study. Exclusion criteria were endoprosthetic knee and hip joints, rheumatoid arthritis, podagra, systemic connective tissue disease, failure to perform functional tests for the study due to significant knee joint disorder or related pathology, sensitive or damaged skin in the area of dirt and/or mineral water, oncologic disease diagnosed or suspected, fewer than 6 months after intraarticular injections, permanent use of pain medication due to comorbidity, pregnancy, pregnancy planning in the near future, refusal, participation in the research, no use of healing lubricants, knee joint, and misunderstanding of the Lithuanian language. The trial involved 95 adults with grade I-III knee joint OA. The subjects were randomly assigned to study groups with a 1:1:1 allocation ratio, according to the rehabilitation registration journal. A total of 92 subjects established the trial, and 3 subjects left the trial because of the following reasons: 1 not meeting inclusion criteria, and 2 subjects refused to participate in the trial (Fig. 1).

Participants of group I (32 subjects) were treated using peat mud applications in the waist and leg area, $36-42{ }^{\circ} \mathrm{C}$ temperature, duration of the procedure $20 \mathrm{~min}, 10$ procedures every other day, specific physical therapy (10 procedures every other day) at sanatorium "Egle," in Druskininkai. Participants of group II (30 subjects) were treated using the mineral sodium chloride bath with mineralization 40-46 g/l, temperature of water $36-38{ }^{\circ} \mathrm{C}$, duration of the procedure $15 \mathrm{~min}, 10$ procedures every other day, specific physical therapy (10 procedures every other day) at sanatorium "Egle," in Druskininkai. Participants of group III (30 subjects), the control group, received specific physiotherapy (10 procedures every other day) at the Hospital of the Lithuanian University of Health Sciences, Rehabilitation Clinic. The physical therapy was standardized in all three groups. All physical therapists had the same protocol with exercises, which was discussed before the study. The subjects received the same exercises, for the same repetition and number of sets, to increase the amplitude of motion of the knee joint, for muscle strengthening and proprioception training. The specific physiotherapy program consisted of 10 procedures every other day and the duration of the procedure was $30 \mathrm{~min}$.

Fig. 1 Trial organization scheme

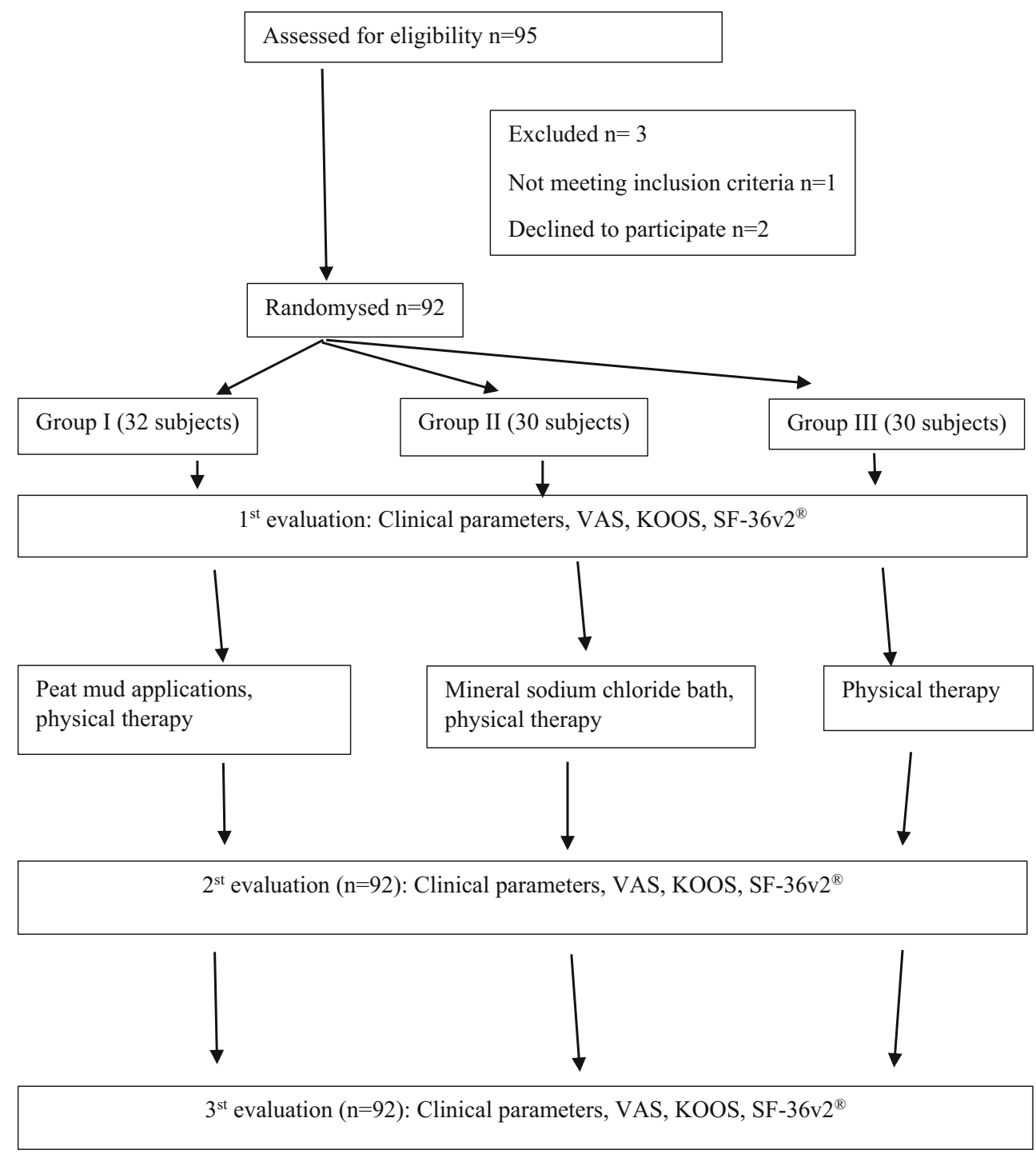


Table 1 The baseline characteristics of subjects (age, sex, body mass index), with respect to groups of subjects

\begin{tabular}{llllll}
\hline Characteristic & $\begin{array}{l}\text { All groups } \\
(n=92)\end{array}$ & $\begin{array}{l}\text { Group I } \\
(n=32)\end{array}$ & $\begin{array}{l}\text { Group II } \\
(n=30)\end{array}$ & $\begin{array}{l}\text { Group III } \\
(n=30)\end{array}$ \\
\hline Age, A (SD) & $64.6(11.4)$ & $65.0(10.8)$ & $61.0(13.4)$ & $67.9(8.9)$ & $p=0.062$ \\
$\begin{array}{l}\text { Sex }(\%) \\
\text { Men }\end{array}$ & 13.0 & 21.9 & 10.0 & 6.7 & $\chi^{2}=3.521 p=0.172$ \\
Women & 87.0 & 78.1 & 90.0 & 93.3 & \\
Body mass index, A (SD) kg/m & $29.4(4.3)$ & $29.3(3.9)$ & $29.2(4.6)$ & $29.8(4.6)$ & $p=0.873$ \\
\hline
\end{tabular}

Group I, provided with bath procedures; group II, provided with mud procedures; group III, control; $A$, average; $S D$, standard deviation; $\chi^{2}$, chi-square criteria; $p$, level of significance.

Physical therapy program consisted of warm up exercises for $5 \mathrm{~min}$ - to improve blood circulation and activate the muscles for the main part. Main part (20 min) physical therapy exercises were made to maintain and improve joint function, mobility, and flexibility. Strengthening exercises were performed in a closed and opened kinematic chain for quadriceps and hamstrings, followed by exercises to strengthen the stabilizing muscles of knee, hip, and ankle joint. Proprioception and gait training were also performed in the main part. Muscle stretching exercises were performed in a cooling down (5 min) part.

The mineral sodium chloride bath, peat mud applications, and physiotherapy were safe and the subjects tolerated them well. In general, the side effect was not observed. The assessors were blinded to the research condition by not being able to check the patient status.

\section{Results}

The study presents the results of the 92 -subject trial. The mean age of the subjects was 64.6 (11.4) years. The majority of the subjects was females $-87.0 \%$, males constituted $13.0 \%$. The mean body weight of subjects was 29.4 (4.3). The baseline characteristics of subjects (age, sex, body mass index), with respect to groups, did not differ significantly (Table 1).

After treatment significantly greater change in the intervention group (group I and group II) was in walking speed, test of 5 sit downs/stand ups flexion range, extension range of the knee of left leg, and flexor and extensor strength of left and right leg muscles, except circumference of right thigh and knee, circumference of left and right calf compared with the control group (Table 2).

Table 2 Distribution of change in clinical parameters after treatment (stages I-II), with respect to groups of subjects

\begin{tabular}{|c|c|c|c|c|}
\hline Variable & Group I $(n=32)$ & Group II $(n=30)$ & Group III $(n=30)$ & $p$ value \\
\hline Walking speed, A (SD), m/s & $0.20(0.25)^{\mathrm{a}}$ & $0.19(0.28)^{\mathrm{b}}$ & $-0.24(0.24)^{\mathrm{a}, \mathrm{b}}$ & $F=27.984,{ }^{\mathrm{a}, \mathrm{b}} p<0.001$ \\
\hline 5 sit downs/stand ups, A (SD), s & $3.83(3.02)^{\mathrm{a}}$ & $4.24(3.79)^{\mathrm{b}}$ & $1.98(2.00)^{\mathrm{a}, \mathrm{b}}$ & $\chi^{2}=11.938,{ }^{\mathrm{a}} p=0.005,{ }^{\mathrm{b}} p=0.002$ \\
\hline \multicolumn{5}{|l|}{ Left leg } \\
\hline Thigh circumference, A (SD), cm & $0.78(1.38)$ & $0.50(1.46)$ & $0.76(1.73)$ & $\chi^{2}=0.921, p=0.631$ \\
\hline Knee circumference, A (SD), cm & $1.42(2.80)$ & $1.40(2.22)$ & $0.96(1.94)$ & $\chi^{2}=1.281, p=0.527$ \\
\hline Calf circumference, A (SD), cm & $1.16(1.17)$ & $1.15(1.29)$ & $0.66(1.50)$ & $F=1.264, p=0.288$ \\
\hline Flexion range, $\mathrm{A}(\mathrm{SD})$, degrees & $16.2(9.3)^{\mathrm{a}}$ & $16.8(14.3)^{\mathrm{b}}$ & $5.4(5.1)^{\mathrm{a}, \mathrm{b}}$ & $\chi^{2}=23.02,{ }^{\mathrm{a}, \mathrm{b}} p<0.001$ \\
\hline Extension range, $\mathrm{A}(\mathrm{SD})$, degrees & $1.92(3.62)$ & $1.0(2.38)^{\mathrm{b}}$ & $1.87(2.17)^{\mathrm{b}}$ & $\chi^{2}=6.309,{ }^{\mathrm{b}} p=0.017$ \\
\hline Flexor strength, A (SD), score & $1.12(0.44)^{\mathrm{a}}$ & $1.04(0.81)^{\mathrm{b}}$ & $0^{\mathrm{a}, \mathrm{b}}$ & $\chi^{2}=51.417,{ }^{\mathrm{a}, \mathrm{b}} p<0.001$ \\
\hline Extensor strength, A (SD), score & $1.20(0.76)^{\mathrm{a}}$ & $1.25(0.85)^{\mathrm{b}}$ & $0.07(0.25)^{\mathrm{a}, \mathrm{b}}$ & $\chi^{2}=42.192,{ }^{\mathrm{a}, \mathrm{b}} p<0.001$ \\
\hline \multicolumn{5}{|l|}{ Right leg } \\
\hline Thigh circumference, $\mathrm{A}(\mathrm{SD}), \mathrm{cm}$ & $1.23(1.75)$ & $1.28(1.80)$ & $0.73(2.16)$ & $\chi^{2}=7.309, p=0.503$ \\
\hline Knee circumference, A (SD), cm & $1.24(1.40)^{\mathrm{a}}$ & $1.12(0.98)^{\mathrm{b}}$ & $0.18(1.58)^{\mathrm{a}, \mathrm{b}}$ & $\chi^{2}=8.807,{ }^{\mathrm{a}} p=0.018,{ }^{\mathrm{b}} p=0.008$ \\
\hline Calf circumference, A (SD), cm & $1.22(2.51)$ & $1.10(1.1)$ & $0.35(1.29)$ & $\chi^{2}=7.348, p=0.132$ \\
\hline Flexion range, $\mathrm{A}(\mathrm{SD})$, degrees & $19.0(13.3)^{\mathrm{a}}$ & $17.2(12.9)^{\mathrm{b}}$ & $5.10(6.40)^{\mathrm{a}, \mathrm{b}}$ & $\chi^{2}=24.127,{ }^{\mathrm{a}, \mathrm{b}} p<0.001$ \\
\hline Extension range, $\mathrm{A}(\mathrm{SD})$, degrees & $2.12(7.14)$ & $1.88(3.79)$ & $2.0(2.33)$ & $\chi^{2}=2.422, p=0.298$ \\
\hline Flexor strength, A (SD), score & $1.17(0.48)^{\mathrm{a}}$ & $1.08(0.40)^{\mathrm{b}}$ & $0^{\mathrm{a}, \mathrm{b}}$ & $\chi^{2}=62.551,{ }^{\mathrm{a}, \mathrm{b}} p<0.001$ \\
\hline Extensor strength, A (SD), score & $1.00(0.80)^{\mathrm{a}}$ & $1.13(0.63)^{\mathrm{b}}$ & $0.07(0.25)^{\mathrm{a}, \mathrm{b}}$ & $\chi^{2}=41.327,{ }^{\mathrm{a}, \mathrm{b}} p<0.001$ \\
\hline
\end{tabular}

Group I, provided with bath procedures; group II, provided with mud procedures; group III, control; $A$, average; $S D$, standard deviation; $F$, by Fisher test; $\chi^{2}$, by Kruskal-Wallis test

a,b $p<0.05$ 
Table 3 Distribution of change of subjects' clinical parameters after 1 month after treatment (stages I-III), with respect to groups of subjects

\begin{tabular}{lllll}
\hline Variable & Group I $(n=32)$ & Group II $(n=30)$ & Group III $(n=30)$ & $p$ value \\
\hline Walking speed, A (SD), m/s & $0.28(0.27)^{\mathrm{a}}$ & $0.24(0.25)^{\mathrm{b}}$ & $-0.20(0.18)^{\mathrm{a}, \mathrm{b}}$ & $F=37.846,{ }^{\mathrm{a}, \mathrm{b}} p<0.001$ \\
5 sit downs/stand ups, A (SD), s & $5.40(3.41)^{\mathrm{a}}$ & $5.61(3.72)^{\mathrm{b}}$ & $2.10(2.61)^{\mathrm{a}, \mathrm{b}}$ & $\chi^{2}=22.603,{ }^{\mathrm{a}, \mathrm{b}} p<0.001$ \\
Left leg & & & \\
Thigh circumference, A (SD), cm & $1.38(2.09)$ & $1.02(1.89)$ & $0.78(1.77)$ & $\chi^{2}=2.082, p=0.353$ \\
Knee circumference, A (SD), cm & $2.0(2.33)$ & $1.67(2.53)$ & $1.11(1.58)$ & $\chi^{2}=2.835, p=0.242$ \\
Calf circumference, A (SD), cm & $1.14(1.50)$ & $1.87(2.50)$ & $0.74(1.55)$ & $\chi^{2}=5.167, p=0.076$ \\
Flexion range, A (SD) degrees & $19.9(10.8)^{\mathrm{a}}$ & $18.8(17.5)^{\mathrm{b}}$ & $4.20(5.50)^{\mathrm{a}, \mathrm{b}}$ & $\chi^{2}=31.944,{ }^{\mathrm{a}, \mathrm{b}} p<0.001$ \\
Extension range, A (SD), degrees & $2.36(4.56)$ & $0.83(2.50)$ & $1.77(2.22)$ & $\chi^{2}=5.393, p=0.067$ \\
Flexor strength, A (SD), score & $1.28(0.54)^{\mathrm{a}}$ & $1.22(0.85)^{\mathrm{b}}$ & $0^{\mathrm{a}, \mathrm{b}}$ & $\chi^{2}=49.015,{ }^{\mathrm{a}, \mathrm{b}} p<0.001$ \\
Extensor strength, A (SD), score & $1.36(0.91)^{\mathrm{a}}$ & $1.30(0.97)^{\mathrm{b}}$ & $0.07(0.25)^{\mathrm{a}, \mathrm{b}}$ & $\chi^{2}=39.506,{ }^{\mathrm{a}, \mathrm{b}} p<0.001$ \\
Right leg & & & \\
Thigh circumference, A (SD), cm & $1.67(1.96)$ & $1.69(2.09)$ & $0.91(2.13)$ & $F=1.282, p=0.283$ \\
Knee circumference, A (SD), cm & $1.80(1.65)^{\mathrm{a}}$ & $1.52(1.53)^{\mathrm{b}}$ & $0.33(1.74)^{\mathrm{a}, \mathrm{b}}$ & $\chi^{2}=13.476,{ }^{\mathrm{a}} p=0.001,{ }^{\mathrm{b}} p=0.004$ \\
Calf circumference, A (SD), cm & $1.34(2.29)$ & $1.50(1.81)$ & $0.36(1.20)$ & $\chi^{2}=5.658, p=0.059$ \\
Flexion range, A (SD), degrees & $22.6(15.9)^{\mathrm{a}}$ & $18.8(15.4)^{\mathrm{b}}$ & $1.13(8.89)^{\mathrm{a}, \mathrm{b}}$ & $\chi^{2}=32.642,{ }^{\mathrm{a}, \mathrm{b}} p<0.001$ \\
Extension range, A (SD), degrees & $2.28(7.59)$ & $2.33(4.46)$ & $1.87(2.97)$ & $\chi^{2}=0.5, p=0.779$ \\
Flexor strength, A (SD), score & $1.24(0.72)^{\mathrm{a}}$ & $1.13(0.54)^{\mathrm{b}}$ & $0^{\mathrm{a}, \mathrm{b}}$ & $\chi^{2}=52.397,{ }^{\mathrm{a}, \mathrm{b}} p<0.001$ \\
Extensor strength, A (SD), score & $1.20(0.96)^{\mathrm{a}}$ & $1.23(0.92)^{\mathrm{b}}$ & $0.07(0.25)^{\mathrm{a}, \mathrm{b}}$ & $\chi^{2}=35.259,{ }^{\mathrm{a}, \mathrm{b}} p<0.001$ \\
\hline
\end{tabular}

Group I, provided with bath procedures; group II, provided with mud procedures; group III, control; $A$, average; $S D$, standard deviation; $F$, by Fisher test; $\chi^{2}$, by Kruskal-Wallis test

a,b $p<0.05$

Table 4 Distribution of the scores of subjects' pain intensity questionnaire before, after treatment, and after 1 month after treatment (I, II, III stage), with respect to groups of subjects

\begin{tabular}{|c|c|c|c|c|}
\hline & $\begin{array}{l}\text { Group I }(n=32) \\
\text { A (SD), score }\end{array}$ & Group II $(n=30)$ & Group III $(n=30)$ & $p$ value \\
\hline \multicolumn{5}{|c|}{ Over the past month } \\
\hline Stage I & $5.88(1.66)$ & $6.33(2.34)$ & $5.67(2.51)$ & \multirow{3}{*}{$\begin{array}{l}F=0.729, p=0.485 \\
\chi^{2}=2.774, p=0.25 \\
F=3.09,{ }^{\mathrm{a}} p=0.044\end{array}$} \\
\hline Stage II & 4.44 (1.93) & $4.57(2.47)$ & $5.27(2.75)$ & \\
\hline Stage III & $3.63(2.04)^{\mathrm{a}}$ & $4.38(2.44)$ & $5.13(2.66)^{\mathrm{a}}$ & \\
\hline \multicolumn{5}{|c|}{ During daytime } \\
\hline Stage I & $5.97(1.91)$ & $6.27(2.10)$ & $4.93(2.52)$ & \multirow{3}{*}{$\begin{array}{l}\chi^{2}=5.024, p=0.081 \\
F=0.406, p=0.668 \\
\chi^{2}=4.934, p=0.085\end{array}$} \\
\hline Stage II & $4.03(2.10)$ & $4.40(2.34)$ & $4.57(2.74)$ & \\
\hline Stage III & $3.25(2.08)$ & $4.48(2.61)$ & $4.57(2.64)$ & \\
\hline \multicolumn{5}{|c|}{ During night time } \\
\hline Stage I & $4.16(2.60)$ & $4.37(3.18)$ & $3.37(3.58)$ & \multirow{3}{*}{$\begin{array}{l}\chi_{2}^{2}=2.939, p=0.23 \\
\chi^{2}=0.953, p=0.621 \\
\chi^{2}=0.6, p=0.741\end{array}$} \\
\hline Stage II & $2.81(2.16)$ & $3.07(2.57)$ & $3.13(3.69)$ & \\
\hline Stage III & $2.34(1.98)$ & $2.55(2.38)$ & $3.0(3.73)$ & \\
\hline \multicolumn{5}{|c|}{ When moving } \\
\hline Stage I & $6.69(2.07)$ & $7.17(2.10)$ & $5.73(2.48)$ & \multirow{3}{*}{$\begin{array}{l}\chi_{2}^{2}=5.553, p=0.062 \\
\chi^{2}=1.172, p=0.556 \\
\chi^{2}=2.194, p=0.334\end{array}$} \\
\hline Stage II & $4.81(2.15)$ & $5.33(2.59)$ & $5.40(2.63)$ & \\
\hline Stage III & $4.25(2.53)$ & $4.72(2.52)$ & $5.20(2.46)$ & \\
\hline \multicolumn{5}{|c|}{ When changing position } \\
\hline Stage I & $6.69(2.49)$ & $6.90(2.35)$ & $6.03(2.48)$ & \multirow{3}{*}{$\begin{array}{l}\chi^{2}=2.371, p=0.306 \\
F=1.948, p=0.149 \\
F=3.325, p=0.485,{ }^{\mathrm{a}} p=0.047\end{array}$} \\
\hline Stage II & $4.66(2.07)$ & $5.0(2.69)$ & $5.90(2.85)$ & \\
\hline Stage III & $4.06(2.50)^{\mathrm{a}}$ & $4.55(2.60)$ & $5.77(2.89)^{\mathrm{a}}$ & \\
\hline \multicolumn{5}{|l|}{ At rest } \\
\hline Stage I & $3.47(2.36)$ & $3.40(2.43)$ & $3.03(3.10)$ & \multirow{3}{*}{$\begin{array}{l}\chi_{2}^{2}=1.299, p=0.522 \\
\chi^{2}=0.967, p=0.617 \\
\chi^{2}=2.319, p=0.314\end{array}$} \\
\hline Stage II & $2.28(2.28)$ & $2.77(2.34)$ & $2.87(3.32)$ & \\
\hline Stage III & $1.50(1.74)$ & $1.83(1.58)$ & $2.93(3.27)$ & \\
\hline
\end{tabular}

Group I, provided with bath procedures; group II, provided with mud procedures; group III, control; $A$, average; $S D$, standard deviation; $F$, by Fisher test; $\chi^{2}$, by Kruskal-Wallis test.

a,b $p<0.05$ 
Table 5 Distribution of the scores of separate areas of SF-36 questionnaire before, after treatment, and 1 month after treatment (stages I, II, III), with respect to groups of subjects

\begin{tabular}{|c|c|c|c|c|}
\hline & $\begin{array}{l}\text { Group I }(n=32) \\
\text { A (SD), score }\end{array}$ & Group II $(n=30)$ & Group III $(n=30)$ & $p$ value \\
\hline \multicolumn{5}{|c|}{ Physical activity } \\
\hline Stage I & $40.2(20.3)$ & $43.4(18.4)$ & $39.5(16.7)$ & $F=0.357, p=0.701$ \\
\hline Stage II & $54.3(23.5)$ & $52.0(16.2)$ & $43.7(16.2)$ & $F=2.552, p=0.084$ \\
\hline Stage III & $53.5(25.1)$ & $55.0(17.2)$ & $45.3(16.3)$ & $F=2.021, p=0.132$ \\
\hline \multicolumn{5}{|c|}{ Restriction of activity due to physical problems } \\
\hline Stage I & $43.0(41.8)$ & $59.2(41.3)$ & $54.3(42.8)$ & $\chi^{2}=2.553, p=0.279$ \\
\hline Stage II & $68.3(35.3)$ & $75.9(32.4)$ & $61.2(38.1)$ & $\chi^{2}=2.072, p=0.355$ \\
\hline Stage III & $68.0(37.7)$ & $72.4(34.9)$ & $60.0(38.1)$ & $\chi^{2}=1.462, p=0.481$ \\
\hline \multicolumn{5}{|l|}{ Pain } \\
\hline Stage I & $58.7(19.3)$ & $52.5(16.8)$ & $58.5(16.5)$ & $\chi^{2}=2.219, p=0.33$ \\
\hline Stage II & $43.4(18.2)$ & $42.8(16.5)$ & $54.4(19.9)$ & $F=2.800, p=0.056$ \\
\hline Stage III & $43.4(21.3)$ & $39.8(17.2)$ & $51.5(22.7)$ & $F=2.492, p=0.086$ \\
\hline \multicolumn{5}{|c|}{ Overall health assessment } \\
\hline Stage I & $55.8(15.7)$ & $52.5(11.4)$ & $50.7(15.7)$ & $F=0.999, p=0.372$ \\
\hline Stage II & $51.2(12.4)$ & $51.2(12.4)$ & $49.3(16.1)$ & $F=0.180, p=0.835$ \\
\hline Stage III & $52.1(13.3)$ & $46.7(12.1)$ & $52.7(16.2)$ & $F=1.611, p=0.206$ \\
\hline \multicolumn{5}{|c|}{ Energy levels/vitality } \\
\hline Stage I & $34.8(6.4)$ & $32.2(8.3)$ & $34.3(11.4)$ & $\chi^{2}=2.172, p=0.338$ \\
\hline Stage II & $35.8(7.4)$ & $32.8(8.4)$ & $33.8(10.8)$ & $\chi^{2}=2.461, p=0.292$ \\
\hline Stage III & $35.8(6.0)$ & $33.6(7.9)$ & $33.5(10.5)$ & $\chi^{2}=1.32, p=0.517$ \\
\hline \multicolumn{5}{|c|}{ Social function } \\
\hline Stage I & $41.5(7.7)$ & $41.9(8.6)$ & $41.5(13.4)$ & $\chi^{2}=0.478, p=0.787$ \\
\hline Stage II & $42.7(8.7)$ & $42.5(6.0)$ & $41.5(13.4)$ & $\chi^{2}=1.291, p=0.524$ \\
\hline Stage III & $42.2(9.4)$ & $41.4(8.3)$ & $43.7(14.6)$ & $\chi^{2}=0.19, p=0.909$ \\
\hline \multicolumn{5}{|c|}{ Restriction of activity due to emotional disorders } \\
\hline Stage I & $72.9(39.2)$ & $75.6(36.0)$ & $74.7(34.1)$ & $\chi^{2}=0.057, p=0.972$ \\
\hline Stage II & $86.2(30.5)$ & $78.2(34.8)$ & $78.2(32.5)$ & $\chi^{2}=3.313, p=0.191$ \\
\hline Stage III & $83.3(33.1)$ & $82.8(32.9)$ & $75.6(34.9)$ & $\chi^{2}=1.887, p=0.389$ \\
\hline \multicolumn{5}{|c|}{ Emotional state } \\
\hline Stage I & $38.1(5.5)$ & $38.3(7.4)$ & $38.0(7.8)$ & $\chi^{2}=0.143, p=0.931$ \\
\hline Stage II & $41.7(7.3)$ & $39.8(8.3)$ & $38.8(8.7)$ & $F=0.985, p=0.378$ \\
\hline Stage III & $40.9(5.7)$ & $37.5(7.7)$ & $40.1(9.1)$ & $\chi^{2}=5.531, p=0.063$ \\
\hline
\end{tabular}

Group I, provided with bath procedures; group II, provided with mud procedures; $A$, average; $S D$, standard deviation; $F$, by Fisher test; $\chi^{2}$, by Kruskal-Wallis test
After 1 month after treatment, all changes of the intervention group (group I and group II) were significantly better, except thigh, knee and calf circumference of the left leg, thigh, and calf circumference left leg, extension range of the left and right leg compared with the control group (Table 3 ).

Based on VAS, after 1 month after treatment (stage III), pain intensity scores over the past month and when changing position were significantly higher in the control group compared with group I (Table 4).

Analysis of separate areas of SF-36 questionnaire shows that the positive changes were identified after treatment and 1 month after the treatment in all areas (Table 5).

On the basis of KOOS questionnaire, during stage I, there was no significant difference between the averages of any subscale of respondents of the intervention group (group I, group II) and the control group (group III). However, after treatment and after 1 month after treatment, average percentages of symptoms, stiffness, and pain of the intervention groups (group I, group II) were significantly better than those of the control group (group III) (Table 6).

The values of parameters of groups I and II, presented in Tables 2, 3, 4, 5, and 6, were not significantly different; therefore, we combined these groups in our prognostic analysis.

For variables with significantly different changes between groups I, II, and control group, we estimated optimal threshold values using ROC test (Table 7). On the basis of binary logistic regression analysis, we predicted chance relations for reaching values greater than optimal for group III subjects. 
Table 6 Distribution of data of KOOS questionnaire on the knee (before, after treatment, and after 1 month after treatment (stages I, II, III), with respect to groups of subjects

\begin{tabular}{|c|c|c|c|c|}
\hline Subscales of KOOS questionnaire & $\begin{array}{l}\text { Group I }(n=32) \\
\text { A (SD), score }\end{array}$ & Group II $(n=30)$ & Group III $(n=30)$ & $p$ value \\
\hline \multicolumn{5}{|l|}{ Symptoms } \\
\hline Stage I & $51.9(15.1)$ & $50.5(13.0)$ & $51.2(17.7)$ & $F=0.068, p=0.934$ \\
\hline Stage II & $66.3(15.3)^{\mathrm{a}}$ & $61.5(16.3)$ & $53.9(20.7)^{\mathrm{a}}$ & $F=3.881,{ }^{\mathrm{a}} p=0.02$ \\
\hline Stage III & $67.2(15.0)^{\mathrm{a}}$ & $64.9(16.8)$ & $53.9(20.7)^{\mathrm{a}}$ & $F=4.929,{ }^{\mathrm{a}} p=0.012$ \\
\hline \multicolumn{5}{|l|}{ Stiffness } \\
\hline Stage I & $55.4(16.3)$ & $53.8(13.4)$ & $49.1(19.8)$ & $F=1.189, p=0.309$ \\
\hline Stage II & $67.2(13.9)^{\mathrm{a}}$ & $64.5(17.4)^{b}$ & $53.4(21.6)^{\mathrm{a}, \mathrm{b}}$ & $F=5.146,{ }^{\mathrm{a}} p=0.009,{ }^{\mathrm{b}} p=0.054$ \\
\hline Stage III & $70.4(15.3)^{\mathrm{a}}$ & $69.0(16.5)^{\mathrm{b}}$ & $53.6(21.7)^{\mathrm{a}, \mathrm{b}}$ & $F=8.112,{ }^{\mathrm{a}} p=0.001,{ }^{\mathrm{b}} p=0.004$ \\
\hline \multicolumn{5}{|l|}{ Pain } \\
\hline Stage I & $53.1(15.7)$ & $48.3(12.5)$ & $47.1(15.4)$ & $F=1.505, p=0.228$ \\
\hline Stage II & $65.3(16.4)^{\mathrm{a}}$ & $60.3(18.3)$ & $51.0(19.1)^{\mathrm{a}}$ & $F=5.023,{ }^{\mathrm{a}} p=0.009$ \\
\hline Stage III & $65.2(14.8)^{\mathrm{a}}$ & $62.2(18.1)$ & $52.3(19.8)^{\mathrm{a}}$ & $F=4.534,{ }^{\mathrm{a}} p=0.014$ \\
\hline \multicolumn{5}{|l|}{ Mobility, everyday life } \\
\hline Stage I & $28.8(17.8)$ & $25.5(15.8)$ & $35.2(21.5)$ & $\chi^{2}=3.548, p=0.17$ \\
\hline Stage II & $40.6(22.3)$ & $42.0(21.4)$ & $39.8(23.2)$ & $F=0.072, p=0.930$ \\
\hline Stage III & $42.8(21.2)$ & $41.8(21.8)$ & $40.2(21.6)$ & $F=0.119, p=0.888$ \\
\hline \multicolumn{5}{|c|}{ Mobility, sports and recreational activities } \\
\hline Stage I & $34.9(16.6)$ & $35.2(20.6)$ & $36.3(15.8)$ & $F=0.048, p=0.953$ \\
\hline Stage II & $44.3(23.7)$ & $46.1(21.6)$ & $39.2(16.8)$ & $F=0.889, p=0.415$ \\
\hline Stage III & $50.2(22.4)$ & $50.0(22.2)$ & $41.3(18.1)$ & $F=1.742, p=0.181$ \\
\hline
\end{tabular}

Group I, provided with bath procedures; group II, provided with mud procedures; group III, control; $A$, average; $S D$, standard deviation; $F$, by Fisher test; $\chi^{2}$, by Kruskal-Wallis test

a,b $p<0.05$

On the basis of non-parametric Spearman analysis, it was found that categorized changes of all parameters presented in Table 7 correlated directly and significantly between themselves.

Distribution of threshold values and their characteristics of KOOS questionnaire's changes of separate scales after treatment and changes 1 month after treatment is presented in Table 8. On the basis of non-parametric Spearman correlation analysis, we found that categorized changes of all parameters presented in Table 8 correlated directly and significantly between themselves.

\section{Discussion}

The positive effect of mineral water and mud therapy is associated with mechanical, thermal, and chemical effect; however, the mechanism of action remains a matter of debate. Kulisch et al. (2014) found the advantage of mineral water baths in reducing pain and improving functional state compared with freshwater therapy. Branco et al. (2016) provide data which shows that sulfur mineral baths (three 20-min baths a week for 10 weeks, at $37-39^{\circ}$ ) are superior in reducing pain and reducing stiffness in the long term compared with freshwater baths. A study which dealt with assessing the effectiveness of mud therapy carried out by Tefner et al. found that both mud packs and specially manufactured thermal packs equally significantly reduced pain, stiffness and improved functional state. Therefore, these authors concluded that the positive impact of mud packs was likely attributable to thermal effect, while chemical effect remained unproven (Tefner et al. 2013). Assessing stiffness and functional state, Güngen et al. (2012) found partial advantage of mud packs compared with thermal packs; meanwhile, assessing pain, both methods had equally significant positive effects. Systematic literature review and metaanalysis published by Antonelli et al. (2018) found statistically significant advantage of a real balneological intervention (thermal mineral water or mud packs), compared with placebo interventions (freshwater or thermal packs), assessing the quality of life of patients with knee joint OA. The results of our study coincide with the results of the aforementioned studies: treatment with mineral sodium chloride mineral baths, peat mud applications together with physiotherapy were more effective than only physiotherapeutic treatment. On the basis of ROC, 
Table 7 Distribution of ROC test predicted values of changes of variables of clinical trials and changes of their characteristics after treatment and 1 month after treatment, according to groups of subjects

\begin{tabular}{|c|c|c|c|c|}
\hline Associated criterion & $\begin{array}{l}\text { AUC } \\
{[95 \% \mathrm{CI}], \%}\end{array}$ & $\begin{array}{l}\text { Sensitivity/specificity } \\
{[95 \% \text { CI }], \%}\end{array}$ & $\begin{array}{l}\text { Group III/group I+II, } \\
n(\%)\end{array}$ & $\begin{array}{l}\text { Group III OR } \\
{[95 \% \mathrm{CI}]}\end{array}$ \\
\hline Walking speed after treatment $\leq 0.025 \mathrm{~m} / \mathrm{s}$ & $94,8[85.4-97.1]$ & $\begin{array}{l}93.3[77.9-99.2] \\
87.1[76.1-94.3]\end{array}$ & $\begin{array}{l}28(93.3) \\
8(12.9)\end{array}$ & $94.5[18.789-475.285]$ \\
\hline Walking speed 1 months after treatment $\leq 0.05 \mathrm{~m} / \mathrm{s}$ & $97.8[92.4-99.8]$ & $\begin{array}{l}93.3[77.9-99.2] \\
96.7[88.7-99.6]\end{array}$ & $\begin{array}{l}28(93.3) \\
2(3.3)\end{array}$ & $413.0[55.287-3085.16]$ \\
\hline 5 sit downs/stand ups after treatment $\leq 1.92, \mathrm{~s}$ & $72.3[61.9-81.2]$ & $\begin{array}{l}69.0[49.2-84.7] \\
77.4[65.0-87.1]\end{array}$ & $\begin{array}{l}20(69.0) \\
14(22.6)\end{array}$ & 7.619 [2.841-20.434] \\
\hline 5 sit downs/stand 1 month after treatment $\leq 2.75 \mathrm{~s}$ & $81.1[71.5-88.6]$ & $\begin{array}{l}65.5[45.7-82.1] \\
86.9[75.8-94.2]\end{array}$ & $\begin{array}{l}19(65.5) \\
8(13.1)\end{array}$ & $12.587[4.33-36.596]$ \\
\hline \multicolumn{5}{|l|}{ Left leg } \\
\hline Flexion range after treatment $\leq 4.0^{\circ}$ & $82.0[71.8-89.8]$ & $\begin{array}{l}56.7[37.4-74.5] \\
91.8[80.4-97.7]\end{array}$ & $\begin{array}{l}17(56.7) \\
4(8.2)\end{array}$ & $14.712[4.207-51.445]$ \\
\hline Flexion range 1 month after treatment $\leq 5^{\circ}$ & 87.4 [77.9-93.8] & $\begin{array}{l}70.0[50.6-85.3] \\
83.3[69.8-92.5]\end{array}$ & $\begin{array}{l}21(70.0) \\
8(16.7)\end{array}$ & $11.667[3.926-34.666]$ \\
\hline \multicolumn{5}{|l|}{ Right leg } \\
\hline Flexion range after treatment $\leq 10.0^{\circ}$ & $82.7[72.6-90.2]$ & $\begin{array}{l}90.0[73.5-97.9] \\
60.0[45.2-73.6]\end{array}$ & $\begin{array}{l}27(90.0) \\
20(40.0)\end{array}$ & $13.5[3.606-50.545]$ \\
\hline Flexion range 1 month after treatment $\leq 12.0^{\circ}$ & 88.0 [78.8-94.2] & $\begin{array}{l}93.3[77.9-99.2] \\
63.3[48.3-76.6]\end{array}$ & $\begin{array}{l}28(93.3) \\
18(36.7)\end{array}$ & $24.111[5.13-113.333]$ \\
\hline \multicolumn{5}{|l|}{ Left leg } \\
\hline Flexor strength after treatment $\leq 0$ score & $93.9[86.1-98.0]$ & $\begin{array}{l}100[88.4-100] \\
89.8[77.8-96.6]\end{array}$ & $\begin{array}{l}30(100) \\
5(10.2)\end{array}$ & - \\
\hline Flexor strength 1 month after treatment $\leq 0$ score & $93.8[85.9-98.0]$ & $\begin{array}{l}100[88.4-100] \\
87.5[74.8-95.3]\end{array}$ & $\begin{array}{l}30(100) \\
6(12.5)\end{array}$ & - \\
\hline \multicolumn{5}{|l|}{ Right leg } \\
\hline Flexor strength after treatment $\leq 0$ score & 98.0 [91.9-99.8] & $\begin{array}{l}100[88.4-100] \\
95.9[86.0-99.5]\end{array}$ & $\begin{array}{l}30(100) \\
2(4.1)\end{array}$ & - \\
\hline Flexor strength 1 month after treatment $\leq .0$ score & $94.9[87.5-98.6]$ & $\begin{array}{l}100[88.4-100] \\
89.8[77.8-96.6]\end{array}$ & $\begin{array}{l}30(100) \\
5(10.2)\end{array}$ & - \\
\hline \multicolumn{5}{|l|}{ Left leg } \\
\hline Extensor strength after treatment $\leq 0$ score & 90.5 [81.8-95.9] & $\begin{array}{l}93.3[77.9-99.2] \\
85.7[72.8-94.1]\end{array}$ & $\begin{array}{l}28(23.3) \\
7(14.3)\end{array}$ & $84.0[16.252-434.166]$ \\
\hline $\begin{array}{l}\text { Extensor strength } 1 \text { month after treatment } \leq 0 \\
\text { score }\end{array}$ & 89.5 [80.5-95.3] & $\begin{array}{l}93.3[77.9-99.2] \\
83.3[69.8-92.5]\end{array}$ & $\begin{array}{l}28(93.3) \\
8(16.7)\end{array}$ & $70.0[13.811-354.781]$ \\
\hline \multicolumn{5}{|l|}{ Right leg } \\
\hline Extensor strength after treatment $\leq 0$ score & $89.7[80.6-95.5]$ & $\begin{array}{l}93.3[77.9-99.2] \\
87.0[73.7-95.1]\end{array}$ & $\begin{array}{l}28(93.3) \\
6(13.0)\end{array}$ & $93.333[17.542-496.59]$ \\
\hline Extensor strength 1 month after treatment $\leq .0$ score & $87.0[77.4-93.6]$ & $\begin{array}{l}93.3[77.9-99.2] \\
78.7[64.3-89.3]\end{array}$ & $\begin{array}{l}28(93.3) \\
10(21 .)\end{array}$ & $51.8[10.505-255.437]$ \\
\hline
\end{tabular}

Group I, provided with bath procedures; group II, provided with mud procedures; group III, control; OR, odds ratio; $C I$, confidence interval; $A U C$, area under the ROC curve

optimal values were estimated for subjects of groups I and II, changes of which significantly differed from control group. On the basis of non-parametric Spearman correlation analysis, changes of presented anthropometric parameters of subjects of all groups and categorized changes of KOOS questionnaire's data correlated between themselves after treatment and 1 month after treatment.

Despite increasing research showing the positive effect of natural factors, on the basis of knee joint OA treatment recommendations provided by international and national experts, the role of these treatment methods remains unclear. SPA therapy is mentioned in EULAR recommendations 2003 as one of the possible non-pharmacological knee joint OA treatment methods. However, updated EULAR recommendations 2013 do not mention SPA therapy (Fernandes et al. 2013;
Jordan et al. 2003). OARSI recommends balneotherapy only for patients with knee joint plus other joint OA as well as with adjacent illnesses; meanwhile, there is a lack of evidence and recommendations for local knee joint OA (McAlindon et al. 2014).

\section{Limitation of the study}

Limitations for this study may include evaluations of longterm (> 12 months) effects and quality of life. Moreover, the patients included in the study were all Kellgren and Lawrence grades 1 to 3 . This is the patients who are willing to take an outpatient rehabilitation program. This is also an unavoidable limitation of our study and the results cannot be generalized to 
Table 8 Distribution of ROC test predicted values of KOOS questionnaire's on the knee changes of variables and changes of their characteristics after treatment and 1 month after treatment, according to groups of subjects

\begin{tabular}{|c|c|c|c|c|}
\hline Associated criterion, score & $\begin{array}{l}\text { AUC }[95 \% \mathrm{CI}], \\
\%\end{array}$ & $\begin{array}{l}\text { Sensitivity/specificity } \\
{[95 \% \text { CI }], \%}\end{array}$ & $\begin{array}{l}\text { Group III/group I+II, } \\
n(\%)\end{array}$ & Group III OR [95\% CI] \\
\hline Symptoms after treatment $\leq 1.5$ & $83.7[74.5-92.8]$ & $\begin{array}{l}86.7[79.7-93.6] \\
80.6[72.6-88.7]\end{array}$ & $\begin{array}{l}26(86.7) \\
12(19.4)\end{array}$ & $27.083[7.942-92.363]$ \\
\hline Symptoms 1 month after treatment $\leq 1.0$ & $82.0[72.7-91.4]$ & $\begin{array}{l}86.7[79.7-93.6] \\
77.4[68.9-86.0]\end{array}$ & $\begin{array}{l}26(86.7) \\
14(22.6)\end{array}$ & $22.286[6.65-74.686]$ \\
\hline Stiffness after treatment $\leq 5.5$ & $76.4[66.2-86.6]$ & $\begin{array}{l}86.7[79.7-93.6] \\
66.1[56.5-75.8]\end{array}$ & $\begin{array}{l}26(86.7) \\
21(33.9)\end{array}$ & $12.69[3.912-41.165]$ \\
\hline Stiffness 1 month after treatment $\leq 5.5$ & $82.8[73.6-92.1]$ & $\begin{array}{l}86.7[79.7-93.6] \\
79.0[70.7-87.4]\end{array}$ & $\begin{array}{l}26(86.7) \\
13(21.0)\end{array}$ & $24.5[7.252-82.769]$ \\
\hline Pain after treatment $\leq 1.5$ & $82.8[73.6-92.1]$ & $\begin{array}{l}86.7[79.7-93.6] \\
79.0[70.7-87.4]\end{array}$ & $\begin{array}{l}26(86.7) \\
13(21.0)\end{array}$ & $24.5[7.252-82.769]$ \\
\hline Pain 1 month after treatment $\leq 1.5$ & $76.3[65.7-86.9]$ & $\begin{array}{l}80.0[71.8-88.2] \\
72.6[63.5-81.7]\end{array}$ & $\begin{array}{l}24(80.0) \\
17(27.4)\end{array}$ & $10.588[3.689-30.389]$ \\
\hline Mobility, everyday life after treatment $\leq 13.5$ & $71.6[60.8-82.3]$ & $\begin{array}{l}86.7[79.7-93.6] \\
56.5[46.3-66.6]\end{array}$ & $\begin{array}{l}26(86.7) \\
27(43.5)\end{array}$ & $8.426[2.625-27.047]$ \\
\hline Mobility, everyday life 1 month after treatment $\leq 24.5$ & $76.2[65.5-87.0]$ & $\begin{array}{l}76.7[68.0-85.3] \\
75.8[67.1-84.6]\end{array}$ & $\begin{array}{l}23(76.7) \\
15(24.2)\end{array}$ & $10.295[3.688-28.736]$ \\
\hline $\begin{array}{l}\text { Mobility, sports and recreational activities after } \\
\text { treatment } \leq 3.0\end{array}$ & $76.4[66.2-86.6]$ & $\begin{array}{l}86.7[79.7-93.6] \\
66.1[79.7-93.6]\end{array}$ & $\begin{array}{l}26(86.7) \\
21(33.9)\end{array}$ & $12.69[3.912-41.165]$ \\
\hline $\begin{array}{l}\text { Mobility, sports and recreational activities } 1 \text { month after } \\
\text { treatment } \leq 3.0\end{array}$ & $79.5[69.3-89.7]$ & $\begin{array}{l}86.7[79.7-93.6] \\
79.0[70.7-87.4]\end{array}$ & $\begin{array}{l}24(80.0) \\
13(21.0)\end{array}$ & $15.077[5.102-44.557]$ \\
\hline
\end{tabular}

all knee OA patients, especially patients with particularly severe functional conditions.

\section{Conclusion}

In the intervention groups of patients with OA of a knee joint, where natural factors were applied (mineral sodium chloride baths and peat mud applications), after treatment and after 1 month after treatment anthropometric data significantly improved, pain intensity and joint stiffness decreased, physical activity increased compared to the control group. Future randomized controlled studies are needed to confirm these results. Moreover, further studies involving a higher number of participants with a longer period of observation are encouraged in order to shed more light on this subject.

Authors' contributions All the authors have reviewed and approved the manuscript before submission.

\section{Compliance with ethical standards}

Conflict of interest The authors declare that they have no conflicts of interest.

Open Access This article is distributed under the terms of the Creative Commons Attribution 4.0 International License (http:// creativecommons.org/licenses/by/4.0/), which permits unrestricted use, distribution, and reproduction in any medium, provided you give appropriate credit to the original author(s) and the source, provide a link to the Creative Commons license, and indicate if changes were made.

\section{References}

Anandkumar S, Nagpal P, Sudarshan S (2014) Efficacy of kinesio taping on isokinetic quadriceps torque in knee osteoarthritis: a double blinded randomized controlled study. Physiother Theory Pract 30(6):375-383 https://www.ncbi.nlm.nih.gov/pubmed/24617598. Accessed 20 Aug 2018

Antonelli M, Donelli D, Fioravanti A (2018) Effects of balneotherapy and spa therapy on quality of life of patients with knee osteoarthritis: a systematic review and meta-analysis. Rheumatol Int [Internet 38(10):1-18. https://doi.org/10.1007/s00296-018-4081-6

Branco M, Rêgo NN, Silva PH et al (2016) Bath thermal waters in the treatment of knee osteoarthritis: a randomized controlled clinical trial. Eur J Phys Rehabil Med 52(4):422-430 https://www.ncbi. nlm.nih.gov/pubmed/26899038. Accessed 26 July 2018

Bruyère $\mathrm{O}$, Cooper C, Pelletier JP, Branco J, Luisa Brandi M, Guillemin F, Hochberg MC, Kanis JA, Kvien TK, Martel-Pelletier J, Rizzoli R, Silverman S, Reginster JY (2014) An algorithm recommendation for the management of knee osteoarthritis in Europe and internationally: a report from a task force of the European Society for Clinical and Economic Aspects of Osteoporosis and Osteoarthritis (ESCEO). Semin Arthritis Rheum 44(3):253-263 https:/www.ncbi.nlm.nih. gov/pubmed/24953861. Accessed 31 July 2018

Campbell TM, Laneuville O, Trudel G (2015) Knee flexion contractures in patients with osteoarthritis: clinical features and histologic characterization of the posterior capsule. PM R 7(5):466-473 https:// www.ncbi.nlm.nih.gov/pubmed/25511691. Accessed 6 Aug 2018

Cutolo M, Berenbaum F, Hochberg M, Punzi L, Reginster JY (2015) Commentary on recent therapeutic guidelines for osteoarthritis. Semin Arthritis Rheum 44(6):611-617 https://www.ncbi.nlm.nih. gov/pubmed/25677861. Accessed 10 Aug 2018

Fernandes L, Bijlsma JWJ, Hagen KB et al (2013) EULAR recommendations for the non-pharmacological core management of hip and knee osteoarthritis. Ann Rheum Dis 72(7):1125-1135 https://www. ncbi.nlm.nih.gov/pubmed/23595142. Accessed 12 Aug 2018 
Fioravanti A, Bender T, Karagülle M et al (2017) Balneotherapy in osteoarthritis: facts, fiction and gaps in knowledge. Eur J Integr Med 9: 148-150 https://hungary.pure.elsevier.com/.../balneotherapy-inosteoarthritis-facts-fiction-and-. Accessed 20 Aug 2018

Güngen G, Ardic F, Findkoglu G et al (2012) The effect of mud pack therapy on serum YKL-40 and hsCRP levels in patients with knee osteoarthritis. Rheumatol Int 32(5):1235-1244 https://www.ncbi. nlm.nih.gov/pubmed/21258804. Accessed 26 Aug 2018

Jordan KM, Arden NK, Doherty M et al (2003) EULAR recommendations 2003: an evidence based approach to the management of knee osteoarthritis: report of a task force of the Standing Committee for International Clinical Studies Including Therapeutic Trials (ESCISIT). Ann Rheum Dis 62(12):1145-1155 https://www.ncbi. nlm.nih.gov/pubmed/14644851. Accessed 30 Aug 2018

Karagülle M, Karagülle MZ (2015) Effectiveness of balneotherapy and spa therapy for the treatment of chronic low back pain: a review on latest evidence. Clin Rheumatol 34(2):207-214 https://www.ncbi. nlm.nih.gov/pubmed/25535198. Accessed 6 Sept 2018

Kraus VB, Blanco FJ, Englund M, Karsdal MA, Lohmander LS (2015) Call for standardized definitions of osteoarthritis and risk stratification for clinical trials and clinical use. Osteoarthr Cartil 23(8):12331241 https://www.ncbi.nlm.nih.gov/pubmed/25865392. Accessed 10 Sept 2018

Kulisch Á, Benkö Á, Bergmann A, Gyarmati N, Horváth H, Kránicz Á, Mándó ZS, Matán Á, Németh A, Szakál E, Szántó D, Szekeres L, Bender T (2014) Evaluation of the effect of Lake Hévíz thermal mineral water in patients with osteoarthritis of the knee: a randomized, controlled, single-blind, follow-up study. Eur J Phys Rehabil Med 50(4):373-381 https://www.ncbi.nlm.nih.gov/pubmed/ 24594851. Accessed 20 Sept 2018

KOOS - Lithuania/Lithuanian - Final version - 02 Mar 2007 - Mapi Research Institute. https:/www.f:/institut/cultadap/project/4061/study4061/final_ versions/kooslitq.doc-02/03/2007. Accessed 6 July 2018

McAlindon TE, Bannuru RR, Sullivan MC et al (2014) OARSI guidelines for the non-surgical management of knee osteoarthritis. Osteoarthr Cartil 22(3):363-388 https://www.ncbi.nlm.nih.gov/ pubmed/24462672. Accessed 14 Sept 2018

Tefner IK, Gaál R, Koroknai A, Ráthonyi A, Gáti T, Monduk P, Kiss E, Kovács C, Bálint G, Bender T (2013) The effect of Neydhartingmud-pack therapy on knee osteoarthritis: a randomized, controlled, double-blind follow-up pilot study. Rheumatol Int 33(10):2569-2576 https://www.ncbi.nlm.nih.gov/pubmed/ 23689969. Accessed 6 Oct 2018

Verhagen AP, Bierma-Zeinstra SMA, Boers M et al (2015) Balneotherapy (or spa therapy) for rheumatoid arthritis. Cochrane Database Syst Rev 11(4):CD000518. https://doi.org/10.1002/14651858. CD000518.pub2 https://www.ncbi.nlm.nih.gov/pubmed/25862243. Accessed 19 Oct 2018

World Health Organization (2013) Chronic rheumatic conditions. Chronic deseases and health promotion. [Internet], Accessed 13 Nov 2016. https://www.who.int/chp/topics/rheumatic/en/. Accessed 21 Oct 2018 